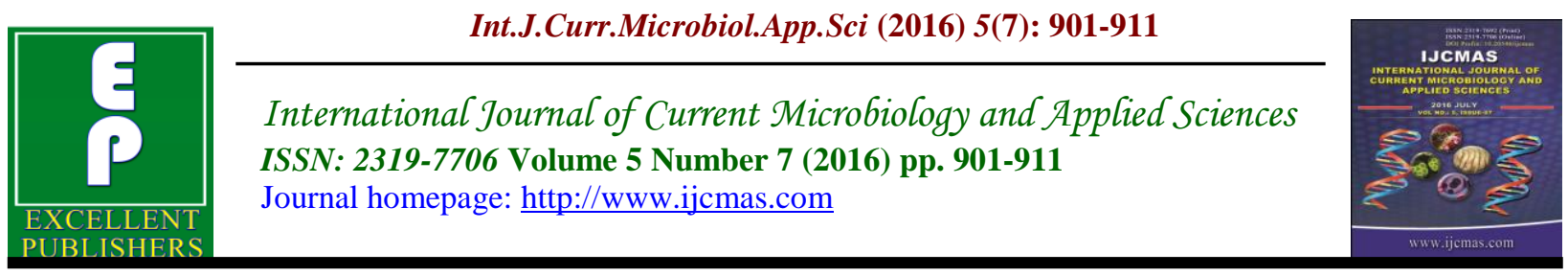

Original Research Article

http://dx.doi.org/10.20546/ijcmas.2016.507.102

\title{
Characterization, Antimicrobial Activity and Antibiotic Susceptibility of Lactic Acid Bacteria Isolated from Food Samples
}

\author{
Priyanka Singh, Pinki Saini*, Shreya Sachan and Shreyasi Dubey \\ Centre of Food Technology, University of Allahabad, India \\ *Corresponding author
}

\section{Keywords}

Isolate, Biochemical characterization, Antibiotic susceptibity, Antimicrobial activity, lactic acid bacteria.

\section{Article Info}

Accepted:

25 June 2016

Available Online:

10 July 2016

\section{A B S T R A C T}

The present study was aimed at isolating Lactobacilli from food samples obtained from local areas of Allahabad. The isolates were biochemically characterized, subjected to Bergy's Manual of Determinative Bacteriology and PIBW in software. They were also tested for antimicrobial activity as well as antibiotic susceptibility. A total of 61 isolates were isolated and identified from 110 food samples. Out of 61 isolates $53.03 \%$ were characterized as L. acidophilus, $8.2 \%$ as L.fermentum, $21.31 \%$ as L.plantarum, $8.2 \%$ as L.casei and $3.2 \%$ were tentatively identified as L.rhamnosus. These results indicate that L.acidophilus is predominant in food samples collected from this sector followed by L.plantarum. All the isolates showed moderate to strong inhibition against S.aureus and Aspergillus strains. Maximum zone of inhibition $(28 \mathrm{~mm})$ was developed by about $59 \%$ of isolates against Aspergillus strain. No inhibition was observed against E.coli strains by any isolate. Antibiotic susceptibility was measured against $2.5 \mathrm{mg} / \mathrm{ml}, 5.0 \mathrm{mg} / \mathrm{ml}$ and $10 \mathrm{mg} / \mathrm{ml}$ concentrations of Ampicillin and Streptomycin. About $47.4 \%$ of isolates were resistant to lower concentrations of Ampicillin $(5 \mathrm{mg} / \mathrm{ml})$ and Streptomycin $(2.5 \mathrm{mg} / \mathrm{ml})$ but became sensitive as the concentration was increased to $10 \mathrm{mg} / \mathrm{ml}$ of both antibiotics. About $44.2 \%$ of isolates were found to be highly sensitive against $10 \mathrm{mg} / \mathrm{ml}$ Ampicillin and Streptomycin.

\section{Introduction}

Lactic Acid Bacteria is the most important group of acid producing bacteria in food industry. The industrial importance of the $\mathrm{LAB}$ is proved by their generally recognized as safe (GRAS) status, because of their contribution to the healthy microflora found in human mucosal surfaces (Patil et al., 2010). They generally contains the genera in the order Lactobacillales, which includes Lactobacillus, Leuconostoc, Lactococcus,Pediococcus and Streptococcus as well as addition to Carnobacterium, Oenococcus, Tetragenococcus, Enterococcus, Vagococcus, and Weisella (Stiles and Holzapfel, 1997). The genus Lactobacillus is very important among the various groups of lactic acid bacteria used in food fermentation and thus have a great economical importance (Schillinger, 1999). They are important part of our intestinal microflora, and their benefits for general state of human health is under serious 
investigation (Salminen et al., 1996). Various species of Lactobacilli are gaining importance in fermentation section of food industry because of their biotechnologically interesting properties (Roy et al., 2000). They are also used as health-promoting probiotic ingredients since they have several therapeutic functions (Oberg et al., 1998) including bile tolerance (Walker and Gilliland, 1983) antibiotic resistance (Curragh and Collins, 1992) and gastric juice tolerance (Kilara, 1982). Lactobacilli consist of a large and diverse group of Gram positive, rod shaped, catalase negative and nonspore forming bacteria which produces lactic acid on fermentation of carbohydrates as the main end product (Pelinescu et al., 2009). The present study has been done to identify and characterize different strains of lactobacillus spp. isolated from different dairy and non dairy food samples.

\section{Material and methods}

\section{Samples collection}

A total of 110 samples including 10 samples each of raw milk, buttermilk, curd, cheese, prebiotics infant formulas, cabbage, cucumber, pumpkin, tomato, carrot, banana, apple were collected from different areas of Allahabad region. The dairy samples collected were both branded and nonbranded in sterile bottles and kept cool until they could be taken to the laboratory. The vegetables and fruit samples were collected from local market. Reference culture of Lactobacillus acidophilus (MTCC 10307) and pathogenic strains (Staphylococcus aureus, Salmonella enterica, Shigella flexenri, E.coli, Aspergillus) for antimicrobial activity was obtained from MTCC, IMTECH, Chandigarh. The bacteriological media and antibiotics were obtained from Hi Media Laboratories Pvt. Ltd., India.

\section{Isolation of Lactic acid bacteria}

The dairy samples were aseptically weighed, homogenized and serially diluted in buffered peptone water. Diluted samples were pour-plated onto Mann Ragosa Sharpe agars (MRS) used for isolating LAB (Badis, et al., 2004; Guessas and Kihal, 2004). The samples of fruits and vegetables were fermented in saline solution for 2 days at room temperature.. Then, $1 \mathrm{~mL}$ of sample was enriched in $9 \mathrm{~mL}$ MRS broth for 1-2 days (Naeem et al., 2012). Subsequently serial dilutions were made using buffered peptone water.To prevent the growth of yeasts, the media were also supplemented with $100 \mathrm{mgL}^{-1}$ of cycloheximide before being incubated at the appropriate temperatures at $37^{\circ} \mathrm{C}$ for $24-48 \mathrm{~h}$. (Beukes et al., 2001; Kalavrouzioti, et al., 2005). Colonies were randomly selected and streak plating was further done to purify the strains(fig 1) which were subsequently kept in two different conditions including at $4^{\circ} \mathrm{C}$ for MRS plates and at $-20^{\circ} \mathrm{C}$ for MRS broths supplemented by $20 \%$ glycerol for further use (Mathara et al., 2004).

\section{Identification of Lactic acid bacteria}

Identification of the isolates were performed according to their morphological, cultural and biochemical characteristics (Mohan kumar and Murugalatha, 2011; Chakraborty and Bhowal, 2015). The morphological characteristics of the isolates such as gram reaction, colour and type of colony , elevation and opacity were studied. The isolates were characterized on the basis of motility test, nitrate reduction test, catalase test, Methyl reduction and Voges- Proskauer test, citrate utilization, indole production in tryptone broth and arginine hydrolysis test as described in Bergy's Manual of systematic Bacteriology (Holt et al., 1994). The identified lactobacillus genus was 
further classified to species level based on their carbohydrate fermentation pattern (Singh and Sharma, 2009).

\section{Physiological characterization of isolates}

Optimum $\mathrm{pH}$ for growth of isolated colonies was determined by incubating $1 \%(\mathrm{v} / \mathrm{v})$ culture into MRS broth at varying $\mathrm{pH}$ ranging from 1-9. For the optimum temperature determination, the cultures were incubated at different temperature $\left(10{ }^{\circ} \mathrm{C}, 37\right.$ ${ }^{\circ} \mathrm{C}, 42{ }^{\circ} \mathrm{C}$ ) under anaerobic conditions. After $24 \mathrm{hr}$ of incubation development of growth was measured by optical density at $560 \mathrm{~nm}$ against a control broth (Chakraborty and Bhowal, 2015). Effect of $\mathrm{NaCl}$ concentration on growth of isolates was also studied, fresh culture $(71 \% \mathrm{v} / \mathrm{v})$ of the isolate was inoculated into MRS broth with a varying $\mathrm{NaCl}$ concentration of $2-10 \%$. After an incubation period of $24 \mathrm{hr}$ the growth was determined by observing the turbidity (Abdulla et al., 2015).

\section{Sugar fermentation pattern}

Different sugars were used for determining the fermentation pattern of lactobacillus genus. Sugars used were Maltose, Arabinose, Fructose, Mannitol, Sorbitol, Sucrose, Lactose. A sugar fermentation broth containing $1 \%$ of respective sugar and inverted Durham's tube was inoculated with isolated strain and incubated at $37^{\circ} \mathrm{C}$ for 24 hrs. The color change and gas production were recorded as positive or negative result. The cultures were further identified on the basis of sugar utilization pattern (Holt et al. 2004; Bhardwaj et al., 2012).

\section{Antimicrobial activity}

Antimicrobial activity of isolated lactobacillus strains were studied against Staphylococcus aureus, Salmonella enterica,
Shigella flexenri, E.coli and Aspergillus using agar well diffusion method (Topisirovic et al., 2006). $500 \mathrm{~mL}$ Erlenmeyer's flasks each containing $200 \mathrm{ml}$ MRS broth was autoclaved at $121^{\circ} \mathrm{C}$ for 15 minutes and inoculated with colony of a LAB isolate grown on MRS agar. The inoculated flasks were incubated at $37^{\circ} \mathrm{C}$ for 2-3 days under stationary condition. Then centrifuged at $10000 \mathrm{rpm}$ for $10 \mathrm{~min}$. After incubation, $2 \mathrm{ml}$ of each fermented culture broth and supernatant was taken to test the antimicrobial activity. The plates were incubated at $37^{\circ} \mathrm{C}$ for $24 \mathrm{hr}$ and inhibition zones were measured.

\section{Antibiotic susceptibility test}

Disk diffusion method was followed to determine the sensitivity of the isolated culture to antibiotics such as Ampicillin and Streptomycin at varying concentrations (2.5$10 \mathrm{mg} / \mathrm{ml}$ ). The test inoculum was prepared by incubating the isolated culture into MRS broth at $37^{\circ} \mathrm{C}$ for $12 \mathrm{hr}$ and $100 \mu \mathrm{l}$ of it was inoculated to Nutrient agar plates by spread plate method. Four wells were made in each of the plates and were filled with $100 \mu \mathrm{l}$ of selected antibiotics each of different concentrations. The plates were then incubated for $24 \mathrm{hr}$ at $37^{\circ} \mathrm{C}$ and the zone of inhibition was measured. (Chakraborty and Bhowal, 2015).

\section{Results and Discussion}

\section{Isolation and identification of Lactobacillus species}

61 colonies were isolated by observing colony morphology, pure cultured and stored in soft agar tube (Table 1). All the colonies were selected on the basis of colony morphology and gram staining. The strains were showing different colonical morphology i.e. colour, size, margin, shape, 
gram reaction and opacity. Isolates obtained from curd samples were found to be gram positive, small in size, creamy white and and shiny in appearance with raised elevation and translucent colony whereas isolates from raw milk samples were also gram positive (fig 2) small transluscent but were white shiny in appearance with convex elevation. About $26 \%$ of the isolates showed large size colonies with creamy white and non-shiny colour and opaque appearance. (Chakarborty and Bhowal, 2015) have also reported that isolated colonies of Lactobacilli showed creamy white colour, circular shape, low convex with entire margins appearance.

Isolated strains of lactobacillus species have shown a negative pattern for catalase activity, nitrate reduction, indole test, motility, arginie hydrolysis and acid from glucose. These are the essential characterisitics of lactobacillus group. However different strains showed variable results in terms of citrate utilization, methyl red and Voge's Proskauer test. The results are in accordance with Pundir et al., (2013), Bhardwaj et al., (2012), (Chakarborty and Bhowal, 2015).

\section{Physiological Characterization}

\section{Growth at different temperature and $\mathrm{pH}$}

The growth of organism was tested against influence of $\mathrm{pH}$ and found to be the highest at $\mathrm{pH} \mathrm{5-7}$ and a little growth at $\mathrm{pH} 8$ and $\mathrm{pH}$ 3. Pundir et al., (2013) have also reported that LAB isolates were able to grow in $\mathrm{pH}$ range 4-7. (Chakarborty and Bhowal, 2015) have also reported that optimum $\mathrm{pH}$ value for Lactic acid bacteria was between 5.5 to 6.5. Hence it can be concluded that lactobacilli can survive in extreme acidic as well as alkaline environment.

Similarly for growth at different temperature all the isolates showed luxuriant growth and turbidity at $37^{\circ} \mathrm{C}$ but little and no growth at $42^{\circ} \mathrm{C}$ and $10^{\circ} \mathrm{C}$ respectively (Table 3 ).

\section{Growth at different $\mathrm{NaCl}$ concentration}

The effect of different $\mathrm{NaCl}$ concentrations $(2,4,6,8$ and $10 \%)$ was observed in LAB in term of turbidity (Table 3). The $4 \% \mathrm{NaCl}$ concentration was found to be optimum for LAB growth. As the concentration was increased from 6-10\%, no turbidity was observed. Pundir et al., (2013) have also reported that Lactic acid bacteria isolates were able to tolerate $1-6.5 \% \mathrm{NaCl}$ concentration.

\section{Sugar Fermentation Pattern}

Different isolates showed different types of sugar utilization patterns (Table 5). On compairing the sugar utilization patterns with those given for Lactobacillus species in the Bergey's Manual of Determinative Bacteriology, the isolates were tentatively identified as L. acidophilus, L. fermentum, L. plantarum, L. casei and L. rhamnosus. The data obtained for genus and species identification comprising a number of morphological, physiological, biochemical and sugar utilization pattern tests were also subjected to software called PIBWin. (Bryant, 2004). Out of 61 isolates $53.03 \%$ were characterized as L. acidophilus, $8.2 \%$ as L.fermentum, $21.31 \%$ as L.plantarum, $8.2 \%$ as L.casei and $3.2 \%$ were tentatively identified as L.rhamnosus (Table 5). Similar use of software for identification of species has been reported by Bhardwaj et al (2012). 
Table.1 Isolation of bacteria from food samples

\begin{tabular}{|l|l|l|l|l|}
\hline Sources & $\begin{array}{l}\text { No. of } \\
\text { samples }\end{array}$ & $\begin{array}{l}\text { No. of } \\
\text { Isolates }\end{array}$ & Codes of Isolates & $\begin{array}{l}\text { Growth on } \\
\text { MRS agar }\end{array}$ \\
\hline Curd & 10 & 9 & C1, C2, C4, C5, C6, C7, C8, C9, C10 & +++ \\
\hline Raw Milk & 10 & 9 & $\begin{array}{l}\text { RM1, RM2, RM3, RM4, RM6, RM7, RM8, } \\
\text { RM9, RM10 }\end{array}$ & +++ \\
\hline Cheese & 10 & 5 & CH3, CH4, CH5, CH6, CH7 & ++ \\
\hline Probiotics & 10 & 3 & Y, Z, F & + \\
\hline Buttermilk & 10 & 7 & BM1, BM2, BM3, BM4, BM5, BM6, BM7 & +++ \\
\hline Apple & 10 & - & & - \\
\hline Banana & 10 & - & & - \\
\hline Cabbage & 10 & 5 & CB1, CB4, CB5, CB6, CB7 & ++ \\
\hline Cucumber & 10 & 8 & Cu1, Cu2, Cu3, Cu4, Cu5,Cu6, Cu7, Cu8 & +++ \\
\hline Carrot & 10 & 7 & Ca3, Ca4, Ca5, Ca6, Ca8, Ca9, Ca10 & +++ \\
\hline Pumpkin & 10 & 8 & P2, P3, P4, P5, P6, P7, P8, P9 & +++ \\
\hline L.acidophilus (MTCC 10307) & & +++ \\
\hline
\end{tabular}

+=Minimum growth; ++=Moderate growth; +++=High growth; - =No growth

Table.2 Morphological characteristics of cultures isolated on MRS agar

\begin{tabular}{|l|l|l|l|l|l|l|}
\hline Isolates & $\begin{array}{l}\text { Gram } \\
\text { Reaction }\end{array}$ & $\begin{array}{l}\text { Type of } \\
\text { colony }\end{array}$ & Color & Margin & Elevation & Opacity \\
\hline C1,C2,C4,C6 & Gram + & Small & $\begin{array}{l}\text { Creamy white, } \\
\text { shiny }\end{array}$ & Entire & Raised & Translucent \\
\hline $\begin{array}{l}\text { RM1,RM2,RM3,RM4, } \\
\text { RM6,RM7,RM8,RM9, RM10 }\end{array}$ & Gram + & Small & White, shiny & Entire & Convex & Translucent \\
\hline C5,C7,C8,C9,C10 & Gram + & Small & Off-white & Entire & Raised & Translucent \\
\hline CB1,CB4,CB5,CB6 & Gram + & Large & $\begin{array}{l}\text { Off-white, non- } \\
\text { shiny }\end{array}$ & Entire & Flat & Opaque \\
\hline Y,Z,F & Gram + & Small & $\begin{array}{l}\text { Off-white, non- } \\
\text { shiny }\end{array}$ & Entire & Flat & Opaque \\
\hline $\begin{array}{l}\text { BM1,BM2,BM3,BM4, BM5, } \\
\text { BM6, BM7,P2 }\end{array}$ & Gram + & Small & $\begin{array}{l}\text { Creamy-white, } \\
\text { non-shiny }\end{array}$ & Entire & Convex & Opaque \\
\hline $\begin{array}{l}\text { Cu1,Cu2,Cu3,Cu4,Cu5, } \\
\text { Cu6,u7,Cu8,Ca5,Ca6,Ca8 } \\
\text { Ca9,Ca10,P3,P4,P5,P6,P7P8, } \\
\text { P9,CB7,CH3,CH4, } \\
\text { CH5,CH6, CH7 }\end{array}$ & Gram + & Large & $\begin{array}{l}\text { Creamy-white, } \\
\text { non-shiny }\end{array}$ & Entire & Flat & Opaque \\
\hline $\begin{array}{l}\text { L.acidophilus(MTCC 10307) } \\
\text { Gram + }\end{array}$ & Large & $\begin{array}{l}\text { Creamy-white, } \\
\text { non-shiny }\end{array}$ & Entire & Flat & Opaque \\
\hline
\end{tabular}


Table.3 Physiological characterization of isolates

\begin{tabular}{|c|c|c|c|c|c|c|c|c|c|c|c|c|c|}
\hline \multirow[b]{3}{*}{$\begin{array}{l}\mathrm{C} 1, \mathrm{C} 2, \mathrm{C} 4, \mathrm{C} 7, \mathrm{C} 8, \mathrm{C} 9, \mathrm{C} 10, \mathrm{RM} 4, \mathrm{R} \\
\text { M6RM7,RM8,BM1,BM2,BM3, } \\
\text { BM4,BM5,Y,Z,F,Ch3,Ch4,Ch5, } \\
\text { Ch6,Ch7Ca3,Ca4,Ca5,Ca6,Ca7, } \\
\text { Ca8,Ca9,Ca10,Cu1,Cu2,Cu3,Cu6, } \\
\text { Cb1,Cb5, Cb6,P3,P4,P5,P7 }\end{array}$} & \multicolumn{3}{|c|}{\begin{tabular}{|l|}
$\begin{array}{l}\text { Effect } \\
\text { temperature }\end{array}$ \\
\end{tabular}} & \multicolumn{5}{|c|}{ Effect of $\mathrm{pH}$} & \multicolumn{5}{|c|}{$\begin{array}{l}\text { Effect } \\
\text { NaCl\% }\end{array}$} \\
\hline & $10^{\circ} \mathrm{C}$ & $37^{\circ} \mathrm{C}$ & $42^{\circ} \mathrm{C}$ & 1 & 4 & 5 & 8 & 9 & 2 & 4 & 6 & 8 & 10 \\
\hline & + & ++ & + & - & + & ++ & - & - & + & ++ & + & - & - \\
\hline $\begin{array}{l}\text { C6,RM1,RM2,RM3,BM6,BM7, } \\
\text { Cb4, Cb7, P6,P8,P9 Cu4,Cu5 }\end{array}$ & - & ++ & + & - & + & ++ & + & - & + & ++ & - & - & - \\
\hline $\mathrm{C} 5, \mathrm{Cu} 7, \mathrm{Cu} 8, \mathrm{RM} 9, \mathrm{RM} 10$ & - & ++ & + & - & - & ++ & - & - & + & ++ & - & - & - \\
\hline L.acidophilus (MTCC 10307) & + & ++ & - & - & + & ++ & - & - & + & ++ & - & - & - \\
\hline
\end{tabular}

$+=$ able to grow; $++=$ maximum growth; $-=$ no growth

Table.4 Biochemical characteristics of isolates

\begin{tabular}{|c|c|c|c|c|c|c|c|c|c|}
\hline Isolates & 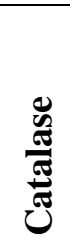 & 窇 & 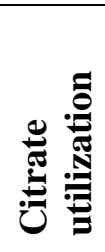 & 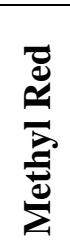 & 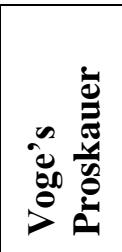 & 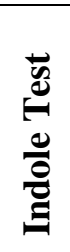 & 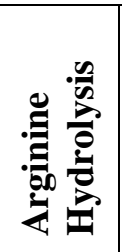 & 总 & 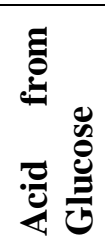 \\
\hline $\begin{array}{l}\text { C1,C2,C4,C5,C8,RM6,RM7,RM8, } \\
\text { RM9,RM10,CH3,CH4,CH5,CH6, } \\
\text { CH7,BM1,BM4,BM5,BM6,BM7, } \\
\text { CB1,CB4,CB5,CB6,CB7,Cu1,Cu3, } \\
\text { Cu4,Cu5,Cu6,Cu7,Cu8,Ca4,Ca9, } \\
\text { Ca10,P5 }\end{array}$ & - & - & + & - & + & - & + & - & + \\
\hline C6,C7,C9,BM2,BM3, & - & - & - & - & + & - & + & - & + \\
\hline $\begin{array}{l}\text { C10,RM3,RM4,Y,Z,F,Cu2,Ca5, } \\
\text { Ca7,P3,P7,P8,P9 }\end{array}$ & - & - & + & - & - & - & + & - & + \\
\hline $\mathrm{Ca} 3, \mathrm{Ca} 6, \mathrm{Ca} 8, \mathrm{P} 4, \mathrm{P} 6$ & - & - & - & + & + & - & + & - & + \\
\hline RM1,RM2 & - & - & - & - & - & - & + & - & + \\
\hline L.acidophilus (MTCC 10307) & - & - & + & - & + & - & + & - & + \\
\hline
\end{tabular}

$+=$ Positive reaction; $-=$ Negative reaction or no reaction 
Table.5 Sugar fermentation pattern by isolates

\begin{tabular}{|c|c|c|c|c|c|c|c|c|}
\hline Isolates & 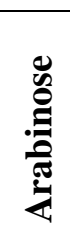 & 苋 & 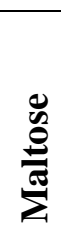 & 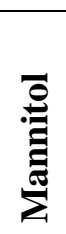 & 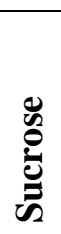 & 苞 & 蒿 & $\begin{array}{l}\text { Probable } \\
\text { Identified } \\
\text { Organism }\end{array}$ \\
\hline $\begin{array}{l}\text { C1,C2,C4,C5,C8,RM6,RM7,RM8, } \\
\text { RM9,RM10,CH3,CH4,CH5,CH6, } \\
\text { CH7,BM1,BM4,BM5,BM6,BM7, } \\
\text { CB1,CB4,CB5,CB6,CB7,Cu1,Cu3 } \\
\text { Cu4,Cu5,Cu6,Cu7,Cu8,Ca4,Ca9, } \\
\text { Ca10,P5 }\end{array}$ & - & + & - & + & + & - & - & $\begin{array}{l}\text { L.acidophilus } \\
(59.03 \%)\end{array}$ \\
\hline $\mathrm{C} 6, \mathrm{C} 7, \mathrm{C} 9, \mathrm{BM} 2, \mathrm{BM} 3$ & $\mathrm{~V}$ & + & + & + & - & - & + & $\begin{array}{l}\text { L.fermentum } \\
(8.2 \%)\end{array}$ \\
\hline $\begin{array}{l}\text { C10,RM3,RM4,Y,Z,F,Cu2,Ca5, } \\
\text { Ca7,P3,P7,P8,P9 }\end{array}$ & $\mathrm{V}$ & + & + & + & + & + & + & $\begin{array}{l}\text { L.plantarum } \\
(21.31 \%)\end{array}$ \\
\hline Ca3,Ca6,Ca8,P4,P6 & - & + & + & + & + & + & - & $\begin{array}{l}\text { L.casei } \\
(8.2 \%)\end{array}$ \\
\hline RM1,RM2 & - & + & + & + & - & + & + & $\begin{array}{l}\text { L.rhamnosus } \\
(3.2 \%)\end{array}$ \\
\hline
\end{tabular}

$\mathrm{V}=$ variable fermentation; + = fermentation; - = no fermentation

Table.6 Antimicrobial activity of isolates

\begin{tabular}{|c|c|c|c|c|c|c|c|}
\hline \multirow[b]{2}{*}{ 菅 } & \multirow[b]{2}{*}{ Isolates } & \multicolumn{6}{|c|}{ Diameter of zone of inhibition zone (DIZ mm) } \\
\hline & & $\begin{array}{l}\tilde{\Xi} \\
\vdots \\
\vdots \\
\vdots \\
0 \\
0 \\
0\end{array}$ & 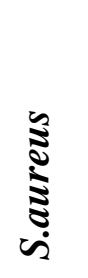 & 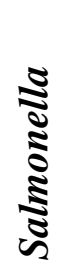 & 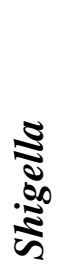 & 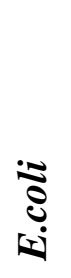 & 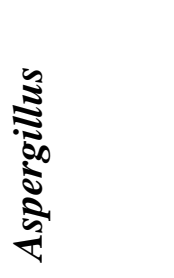 \\
\hline I & $\begin{array}{l}\mathrm{C} 1, \mathrm{C} 2, \mathrm{C} 4, \mathrm{C} 5, \mathrm{C} 8, \mathrm{RM} 6, \mathrm{RM} 7, \mathrm{RM} 8, \\
\text { RM9,RM10,CH3,CH4,CH5,CH6, } \\
\text { CH7,BM1,BM4,BM5,BM6,BM7,CB1 } \\
\text { CB4,CB5,CB6,CB7,Cu1,Cu3,Cu4, } \\
\text { Cu5,Cu6,Cu7,Cu8,Ca4,Ca9,Ca10,P5 }\end{array}$ & $59 \%$ & $\begin{array}{l}\text { SI } \\
15\end{array}$ & $\begin{array}{l}\text { MI } \\
4\end{array}$ & $\begin{array}{l}\text { MI } \\
6\end{array}$ & NI & $\begin{array}{l}\text { VSI } \\
28\end{array}$ \\
\hline II & $\begin{array}{l}\text { C6,C7,C9,BM2,BM3C10,RM3,RM4 } \\
\text { Y,Z,F,Cu2,Ca5,Ca7,P3,P7,P8, P9 }\end{array}$ & $29.5 \%$ & $\begin{array}{l}\text { SI } \\
18\end{array}$ & $\begin{array}{l}\text { MI } \\
2\end{array}$ & $\begin{array}{l}\text { MI } \\
5\end{array}$ & NI & $\begin{array}{l}\text { SI } \\
15\end{array}$ \\
\hline III & $\mathrm{Ca} 3, \mathrm{Ca} 6, \mathrm{Ca} 8, \mathrm{P} 4, \mathrm{P} 6$ & $8.19 \%$ & SI 5 & NI & NI & NI & SI 10 \\
\hline IV & RM1,RM2 & $3.27 \%$ & MI4 & NI & NI & NI & MI5 \\
\hline $\mathrm{V}$ & L. acidophilus (MTCC 10307) & - & 22 & 8 & 11 & 5 & 28 \\
\hline
\end{tabular}

$\mathrm{VSI}=$ very strong inhibition; $\mathrm{SI}=$ strong inhibition; $\mathrm{MI}=$ moderate inhibition;

$$
\mathrm{NI}=\text { no inhibition }
$$


Table.7 Antibiotic susceptibility of isolates

\begin{tabular}{|c|c|c|c|c|c|c|c|c|}
\hline \multirow{3}{*}{$\stackrel{0}{0}$} & \multirow[t]{3}{*}{ Antibiotics } & \multirow{3}{*}{ 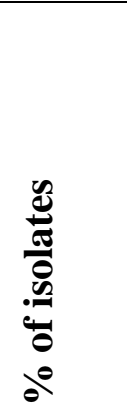 } & \multicolumn{6}{|c|}{$\begin{array}{l}\text { Diameter of zone of inhibition zone } \\
\text { (DIZ mm) }\end{array}$} \\
\hline & & & \multicolumn{3}{|c|}{$\begin{array}{l}\text { Ampicillin } \\
\text { (in mg/ml) }\end{array}$} & \multicolumn{3}{|c|}{$\begin{array}{l}\text { Streptommycin } \\
\text { (in } \mathrm{mg} / \mathrm{ml} \text { ) }\end{array}$} \\
\hline & & & 2.5 & $\mathbf{5 . 0}$ & 10 & 2.5 & 5.0 & $\mathbf{1 0}$ \\
\hline $\mathrm{I}$ & $\begin{array}{l}\text { C1,C2,C4,C5,C8,RM6,RM7,RM8, } \\
\text { RM9,RM10,CH3,CH4,CH5,CH6, } \\
\text { CH7,BM1,BM4, BM5, BM6, BM7, } \\
\text { CB1,CB4,CB5,CB6,CB7, Cu1,Cu3, }\end{array}$ & $44.2 \%$ & 5 & 10 & 12 & 4 & 8 & 15 \\
\hline II & $\begin{array}{l}\mathrm{Cu} 4, \mathrm{Cu} 5, \mathrm{Cu} 6, \mathrm{Cu} 7, \mathrm{Cu} 8, \mathrm{Ca} 4, \mathrm{Ca} 9, \\
\mathrm{Ca} 10, \mathrm{P} 5, \mathrm{C} 6, \mathrm{C} 7, \mathrm{C} 9, \mathrm{BM} 2, \mathrm{BM} 3, \mathrm{C} 10, \\
\text { RM3,RM4 Y,Z,F,Cu2,Ca5 }\end{array}$ & $36 \%$ & NI & NI & 5 & NI & 5 & 10 \\
\hline III & Ca3,Ca6,Ca8,P4,P6,RM1,RM2 & $11.4 \%$ & NI & NI & 5 & NI & 10 & 15 \\
\hline IV & L.acidophilus(MTCC 10307) & - & 6 & 12 & 16 & 8 & 12 & 19 \\
\hline
\end{tabular}

Fig.1 Isolation of Lactobacillus spp. from food samples

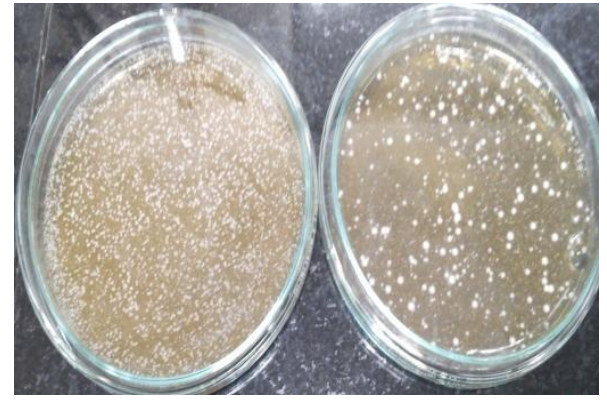

plating of samples

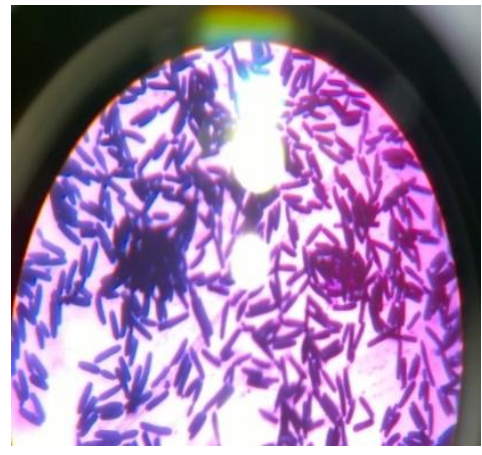

Gram Staining

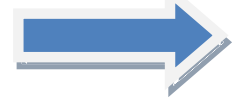

streaking of single colony

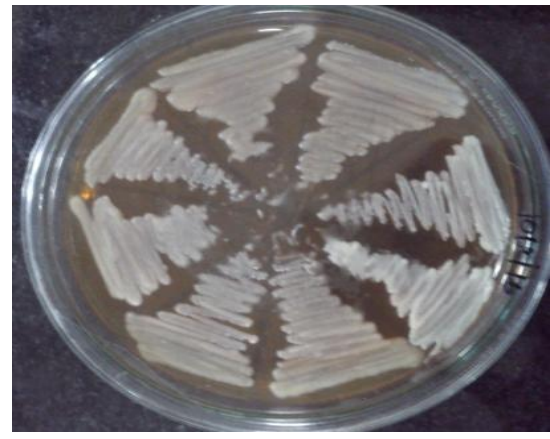

Purified colony 
Fig.2 Gram Staining: (a) Buttermilk, (b) Raw Milk, (c) Pumpkin

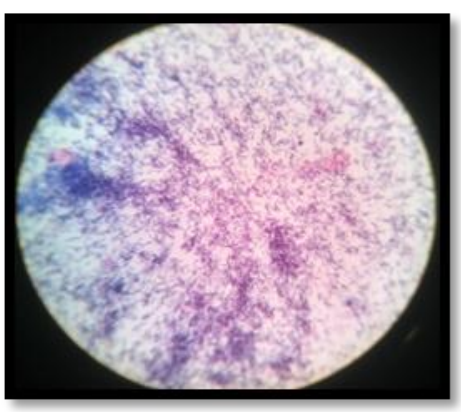

(a)

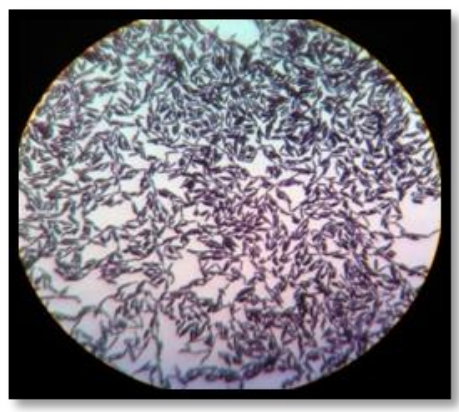

(b)

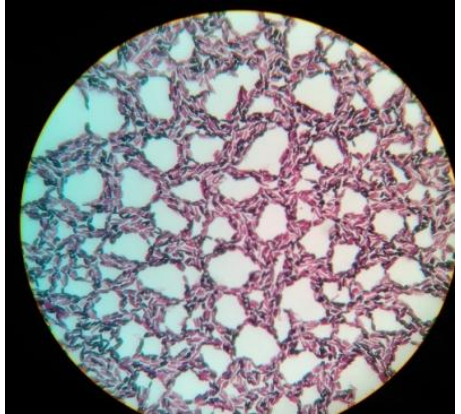

(c)

Fig.3 Prevalence of Lactobacillus strains

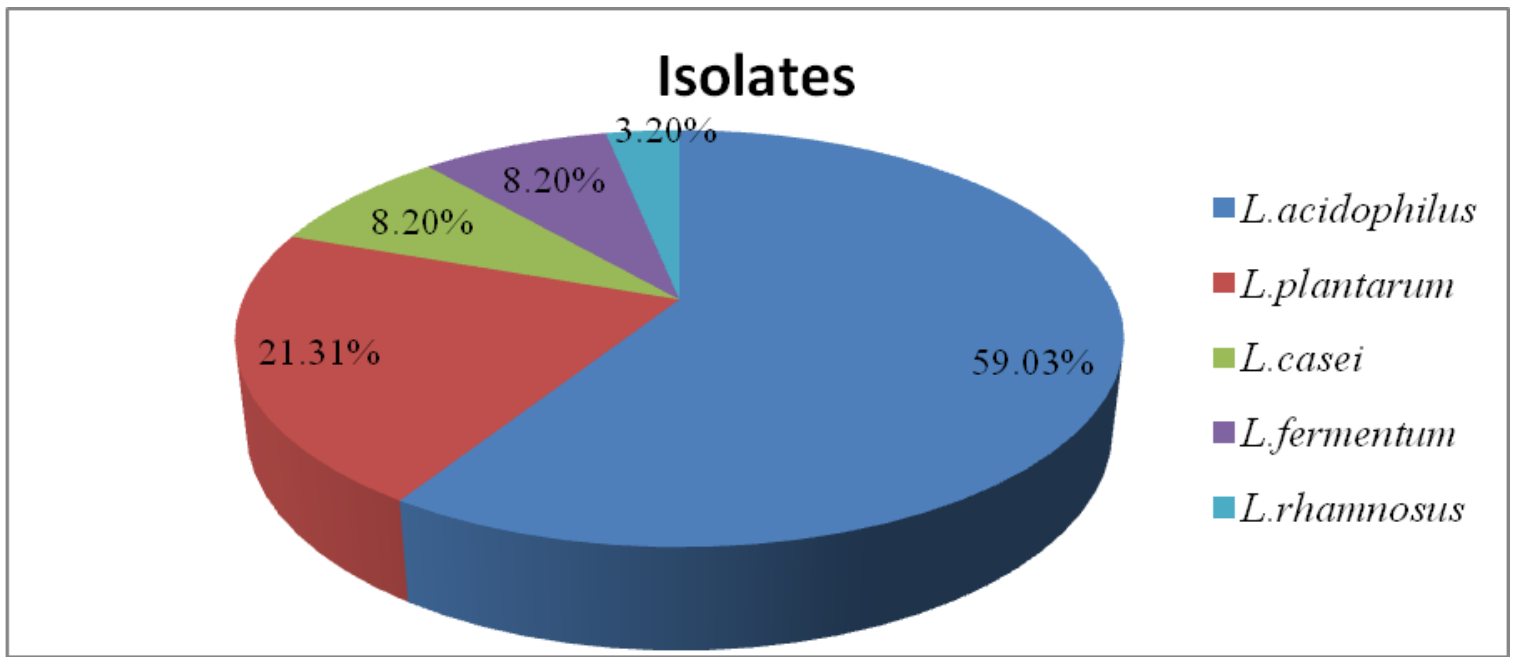

\section{Antimicrobial Activity of isolates}

Table 6 represents the antimicrobial effect of the isolated strains against different pathogenic microorganism. The antimicrobial activities exhibited by Lactobacillus species indicates that the cell free solution of isolated Lactobacillus species were able to inhibit the growth of all the test microorganisms except E. coli. Maximum inhibition zone $(28 \mathrm{~mm})$ was observed against Aspergillus strain followed by S.aureus (18mm). Pundir et al., (2013) have also reported that Lactobacillus showed maximum zone of inhibition (31mm) against Aspergillus whereas minimum zone of inhibition was observed against E.coli and Salmonella.

\section{Antibiotic susceptibility of isolates}

The antibiotic susceptibility of the isolates against different concentrations of Ampicillin and Streptomycin is given in Table 7 . About $44.2 \%$ of isolates were found to be highly sensitive against $10 \mathrm{mg} / \mathrm{ml}$ Ampicillin and Streptomycin but about $47.4 \%$ of isolates were resistant to lower concentrations of Ampicillin $(5 \mathrm{mg} / \mathrm{ml})$ and Streptomycin (2.5mg/ml). Zhou et al. (2005) has also reported high resistance of Lactobacilli to streptomycin. The findings 
are in accordance with previously reported results of Belleti et al., 2009, where most of the tested L.acidophilus and L.plantarum strains were susceptible to Ampicillin and Streptomycin.

In conclusion, on the present study, a total of 61 isolates were obtained from dairy and vegetables sample. The isolates were identified as L. acidophilus, L. fermentum, L. plantarum, L.casei and L. rhamnosus $L$. acidophilus was the most prevelant species in the samples studied. The strains were found to have antimicrobial activity against pathogens except $E$. coli. The isolates were resistant to lower concentrations of antibiotics studied but as the concentration was increased the isolated strains become susceptible. These characteristics shows that the isolates have potential to be used as a natural preservative and for probiotic applications.

\section{Acknowledgement}

Authors are thankful to MTCC, IMTECH, Chandigarh for providing necessary strains required for the research.

\section{References}

Abdulla, A.A., Thikra, A.A., Anwar, K., Saffar, A.L., Alahmed, S.G., Saffar, H.K.A.L., Wesawei, Y.A. 2015. Analysis of Probiotic properties of Lactobacillus acidophilus from commercial yoghurt. Int. J. Curr. Microbiol. App. Sci., ISSN: 23197706 Volume 4 Number 12 pp. 5485544(12): 548-554.

Badis, A., Guetarni, D., Moussa- Boudjenea, B., Henni, D.E., Tornadijo, M.E., Kihal, M. 2004. Identification of cultivable lactic acid bacteria isolated from Algerian raw goat's milk and elevation of their technological properties. Food Microbiol., 21: 343-349.

Belletti, N., Gatti, M., Bottari, B., Neviani, E., Tabanelli, G., Gardini, F. 2009. Antibiotic resistance of Lactobacilli isolated from two Italian hard cheeses. J. Food Protection, 72(10): 2162-2169.

Bukas, E.M., Bester, B.H., Mostert, J.F. 2001. The microbiology of south African traditional fermented milks. Int. J. Food Microbiol., 63: 189-197.

Bhardwaj, A., Monica Puniya, M., Sangu, K.P.S., Kumar, S., Dhewa, T. 2012. Isolation and Biochemical Characterization of Lactobacillus species Isolated from Dahi. J. Dairy Sci. Technol.

Bryant, T.N. 2004. J. Appl. Microbiol., 97(6): 1326-1327p.

Chakraborty, A., Bhowal, J. 2012. Isolation, Identification and Analysis of Probiotic Properties of Lactobacillus Spp. from Selected Regional Dairy Product. Int. J. Curr. Microbiol. Appl. Sci., ISSN: 23197706 Volume 4 Number 6 (2015) pp. 621628

Curragh, H.J,. Collins, M.A. 1992. High levels spontaneous drug resistance in Lactobacillus. J. Appl. Bacteriol., 73: 3136.

Guessas, B., Kihal, M. 2004. Characterization of Lactic acid bacteria isolated from Algerian arid zone raw goats milk. African J. Biotechnol., 3: 339-342.

Hertel, C., Ludwig, W., Pot, B., Kersters, K. and Schleifer, K.H. 1993. Differentiation of lactobacilli occurring in fermented milk products by using oligonucleotide probes and electrophoretic protein pro®les. Systematic and Appl. Microbiol., 16: 463467.

Holt, J.G., Kreig, N.R., Sneath, P.H.A. 2004. Bergey's Manual of Determinative Bacteriology.

Kalavrouzioti, I., Hatzikamari, M, LitopoulouTzanetaki, E., Tzanetakis, N. 2005. Production of hard cheese from caprine milk by the use of two types of probiotic cultures as adjuncts. Int. J. Dairy technol., 58: 30-38.

Kilara, A. 1982. Influence of in vitro gastric digestion on survival of somelacticcultures. Milchhwis-senschaft, 37: 129-132.

Mahrosh Naeem, M., Ilyas, M., Haider, S., Baig, S., Saleem. 2012. Isolation 
Characterization and Identification of Lactic acid bacteria from fruit juices and their efficacy against antibiotics. Pak. J. Bot., 44: 323-328.

Mathara, Schillinger, Kutima, Mbugua, S.K., Holzapfel. 2004. Isolation, identification and characterization of the dominant microorganisms of kule naoto: The Maasai traditional fermented milk in Kenya. Int. J. Food Microbiol., 94: 26727.

Milliere, J., Abidi, F., Lefebre, G. 1996. Taxonomic characterization of Lactobacillus delbrueckii subspecies bulgaricus isolated from Cameroonian zebu's fermented raw milk. J. Appl. Microbiol., 80: 583-588.

Milliere, J., Abidi, F. and Lefebre, G. 1996. Taxonomic characterization of Lactobacillus delbrueckii subspecies bulgaricus isolated from Cameroonian zebu's fermented raw milk. J. Appl. Microbiol., 80: 583-588.

Oberg, C.J., Broadbent, J.R., Mcmahon, D.J. 1998. Applications of EPSproduction by LAB. J. Appl. Microbiol., 150: 11871193.

Pelinescu, D.R., Sasarmaan, E., Chifiriu, M.C., Staca, I., Nohita, A.M., Avram, I., Serbancea, F., Dimov, T.V. 2009. Isolation and identification of some Lactobacillus and Enterococcus by a polyphasic taxonomical approach Romaniun Biotechnol. Lett., 14: 42254233.

Pundir, R.K., Rana, S., kashyap, N., Kaur, A. 2013. Probiotic Potential of Lactic acid bacteria Isolated from food Samples - an in Vitro study. J. App. Pharm. Sci., 3(03): 085- 093.

Roy, P., Ward, D., Vincent, F. Mondou. 2000. Molecular identification of potentially probiotic lactobacilli. Curr. Microbiol., 40: 40-46.

Salminen, S., Isolauri, E., Salminen, E.1996. Clinical uses of probiotics for stabilizing the gut mucosal barrier: successful strains and future challenges. Antonie van Leeuwenhoek . 70: 347-358.

Salminen, S.C., Bouley, M.C., Boutron-Ruault, J.H. 1998.Cummings, Franck A, Gibson GR, Isolauri E, Moreau MC, Roberfroid M, Rowland I. Functional food science and gastrointestinal physiology and function. British J. Nutri., 80: 147-171.

Singh, G.P., Sharma, R.R. 2009. Asian J. Exp. Sci., 23(1): 173-179.

Stiles, M.E., Holzapfel, W.H. 1997. Review article: Lactic acid bacteria of foods and their current taxonomy. Int. J. Food Microbiol. 36: 1-29.

Topisirovic, L., Kojic, M., Fira, L., Golic, N., Strahinic, I., Lozo. J. 2006. Potential of lactic acid bacteria isolated from specific natural niches in food production and preservation. Int. J. Food Microbiol., 112: 230-235.

Walker, D.K., Gilliland, S.E. 1983. Relationship among bil tolerance, bile salt deconjugation and assimilation of cholesterol by Lactobacillus acidophilus. J. Dairy Sci., 76: 956-961.

Zhou, J.S., Pillidge, C.J., Gopal, P.K., Gill. 2005. Antibiotic susceptibility profiles of new probiotic Lactobacillus and Bifidobacterium strains. Int. J. Food Microbiol., 98: 211-217.

\section{How to cite this article:}

Priyanka Singh, Pinki Saini, Shreya Sachan and Shreyasi Dubey. 2016. Characterization, Antimicrobial Activity and Antibiotic Susceptibility of Lactic Acid Bacteria Isolated from Food Samples. Int.J.Curr.Microbiol.App.Sci. 5(7): 901-911. doi: http://dx.doi.org/10.20546/ijcmas.2016.507.102 